\title{
Temperature dependence of plasmon resonances in spheroidal metal nanoparticles
}

\author{
N.I. Grigorchuk \\ Bogolyubov Institute for Theoretical Physics of the National Academy of Sciences of Ukraine, \\ 14-b Metrologichna St., 03680 Kyiv, Ukraine
}

Received May 30, 2013, in final form July 1, 2013

The effect of the electron temperature on both the light absorption and the scattering by metal nanoparticles (MNs) with excitation of the surface plasmon electron vibrations is studied in the framework of the kinetic theory. The formulae for electroconductivity and polarizability tensors are derived for finite temperatures of an electron gas. The electrical conductivity and the halfwidth of the surface plasmon resonance are studied in detail for a spherical MN. Depending on the size of MN, the efficiencies of light absorption and scattering with the temperature change are investigated. It is found, in particular, that the absorption efficiency can both increase and decrease with a temperature drop. The derived formulas make it possible to analytically calculate various optical and transport phenomena for MNs of any spheroidal shape embedded in any dielectric media.

Key words: electron temperature, metal nanoparticles, electroconductivity, polarizability tensor, surface plasmon resonance

PACS: 78.67.-n, 65.80.-g, 73.23.-b, 68.49.Jk, 52.25.0s

\section{Introduction}

When the metal nanoparticle is illuminated with a laser beam by frequency which coincides with the frequency of collective electron oscillations in the MN, the surface plasmon resonance (SPR) is excited. The frequency and the width of SPR depends on the size, morphology, spatial orientation of MN and on the dielectric environment [1, 2]. Resonance light scatterers are employed in various applications ranging from surface enhanced Raman scattering [3], near-field scanning optical microscopes [4], to biochemical imaging [5], surface enhanced fluorescence [6], subwavelength optical waveguides [7], cancer therapy [8] etc.

The temperature effect on the optical and transport properties of the metal nanoparticles is very important for pure and applied science of nanoparticles [9]. Since a MN absorbs laser energy in a thinsurface layer, rapid local heating can occur at the surface. Thus, for a detailed analysis of the laser-light absorption or reflection at MN surfaces, the properties of MNs at electron temperatures must be studied. The temperature dependence of the SP resonance is crucial due to a number of recent applications of noble MNs in computer chips [10], thermally assisted magnetic recording [11], thermal cancer treatment [12], catalysis and nanostructure growth [13]. The use of nano-objects as temperature sensors [14] and thermometers [15] is quite promising due to their small sizes and short thermal relaxation time.

Previous calculations of the effect of the temperature on different properties of the MNs were performed in the zero-temperature limit [16, 17] or in the interval $15 \div 1000 \mathrm{~K}$ only [9, 18- 30 ]. While the optical properties of both the bulk metals [31-35] and the MNs in the low-temperature regime are now well studied, the understanding of the effects of electron temperature (typically of the order of $10^{4} \mathrm{~K}$ ) on the plasmon modes has remained a challenge and is not so well understood as at the $T=0$. Such an out-of-equilibrium situation can easily be induced in metallic nanoparticles [36] using ultrashort optical pulses. At time $t=0$, only the electrons are excited by the laser pulse; then the excited electrons would decay through light radiation, phonon excitation, and/or electron-electron scattering. In the most commonly used approach, the two-temperature model [34, 37, 38], assumes that both electronic and ionic 
degrees of freedom are in thermal equilibrium conditions individually, but each degree of freedom has its own temperature. The energy exchange between the electrons and the lattice in this model leads to a time-dependent electronic temperature $T_{\mathrm{e}}(t)$.

The effect of electron temperature was not studied in detail so far because a broad temperature interval requires the use of materials with high thermal stability. In [22-25, 30], an improved Drude model was used where the electron concentration and/or a plasma frequency are directly dependent on the electron temperature. Only a few works have addressed this problem so far [39, 40].

In this work, we focus on optical properties of metal nanoparticles at relatively high electron temperatures, whereas the ion temperature, $T_{\mathrm{i}}$, is supposed to be much smaller [36]. Different behavior with temperature of optical lines for MNs with different radii and shapes is a particular motivation for our present study. The screening coming from the surrounding matrix is taken into account through a constant dielectric function, $\epsilon_{\mathrm{m}}$.

To calculate the effect of the electron temperature on the optical and transport characteristics of MNs, we use the kinetic theory method which accounts for the electron scattering on the MN boundary. It is shown that the efficiency of absorption with temperature strongly depends on the size of MN. It is predicted that the effect of temperature on the MNs with different radii can substantially modify their optical properties. We present our theoretical results on the temperature dependence of the conductivity and polarizability tensors related to the MNs having a spheroidal shape.

The rest of the paper is organized as follows. The Boltzmann equation approach to the problem is presented in section 2. Section 3 contains the study of conductivity tensor in the spheroidal MNs at finite temperatures. In section 4 , we consider the effect of temperature on the polarizability of MN. Section 5 is devoted to the study of light absorption and scattering crossection by MNs. The discussion of the obtained results is available in section 6 , while section 7 contains the conclusions.

\section{Boltzmann equation}

To account for the temperature effect on the conductivity and polarization properties of the MNs, we use the Boltzmann equations approach. The advantage of this approach is that the obtained results can be applied not only to MNs with a spherical shape, but to strongly anisotropic spheroidal (needle-like or diskshaped) MNs. Thus, it permits to study the effect of the particle shape on the physical values measured. Second, the Boltzmann equation method enables us to investigate the MNs having sizes smaller than the electron mean free path $l$. But in the case of sizes less than $l$, it provides the same results as known from other approaches.

There exists a lower limit of applicability of Boltzmann method in a small radius limit when the particle size is comparable to the de Broglie wavelength of the electron, and the quantization of the electron spectrum starts to play an essential role [41]. Practically, it is around a radius greater or less than $2 \mathrm{~nm}$.

Let us consider a single ellipsoidal metal $\mathrm{MN}$ with semiaxes $a, b, c$ that is irradiated by an electromagnetic (EM) wave whose electric field is given as

$$
\mathbf{E}=\mathbf{E}_{0} \exp [\mathrm{i}(\mathbf{k} \cdot \mathbf{r}-\omega t)]
$$

Here, $\mathbf{E}_{0}$ is the amplitude of an electric field of a pump laser, $\omega$ is its frequency, $\mathbf{k}$ is the wave vector, and $\mathbf{r}$ and $t$ describe the spatial coordinates and time.

We restrict ourselves to the case of Rayleigh scattering, where the electromagnetic wavelength (from a pump laser) $\lambda \sim c / \omega$ is much larger than the diameter of the nanoparticle $d(=\max \{a, b, c\})$. Then, the electromagnetic field around the MN can be considered as homogeneous. Placing the coordinate origin in the center of the particle, the above-mentioned assumption is written as follows:

$$
k r \ll 1 .
$$

The inequality (2.2) implies that the $\mathbf{E}$ field of the electromagnetic wave can be considered to be spatially uniform on scales of the order of a particle size such that all the conduction electrons move in-phase producing only dipole-type oscillations. The amplitude of such a field is linked to $\mathbf{E}_{0}$ by the relation [42]

$$
E_{0}^{(j)}(0, \omega) / E_{\mathrm{in}}^{(j)}(\omega)=1+L_{j}\left[\epsilon(\omega) / \epsilon_{\mathrm{m}}-1\right],
$$


where $\epsilon(\omega)$ is the dielectric permittivity of the $\mathrm{MN}, \epsilon_{\mathrm{m}}$ is the dielectric constant of the adjacent medium (i.e., solvent), and $L_{j}$ are depolarization factors in the $j$-th direction (in the principal axes of an ellipsoid).

The electric field $E_{\text {in }}$ gives rise to high-frequency current inside the MN. To obtain the average density of this current over the MN, it is necessary first of all to calculate the electron velocity distribution function. The field $E_{\text {in }}$ has an effect on the equilibrium electron velocity distribution and thus determines the appearance of a nonequilibrium addition $f_{1}(\mathbf{r}, \mathbf{v}, t)$ to the Fermi distribution function

$$
f_{0}(\varepsilon)=\frac{1}{\exp \left[\frac{\varepsilon-\mu}{k_{\mathrm{B}} T}\right]+1} .
$$

Here, $\mu$ is the chemical potential, $k_{\mathrm{B}}$ is Boltzmann's constant, $\varepsilon=m v^{2} / 2$ denotes the kinetic energy of an electron, $v=|\mathbf{v}|$ refers to the electron velocity, and $m$ is the electron mass. As is well known [43, 44], the equilibrium function $f_{0}(\varepsilon)$ does not give any input to the current. Accounting for both the time dependence of equation (2.1) and the inequality (2.2), the total distribution function of electrons can be represented as follows:

$$
f(\mathbf{r}, \mathbf{v}, t)=f_{0}(\varepsilon)+f_{1}(\mathbf{r}, \mathbf{v}, t) \equiv f_{0}(\varepsilon)+f_{1}(\mathbf{r}, \mathbf{v}) \mathrm{e}^{\mathrm{i} \omega t} .
$$

We seek the function $f_{1}(\mathbf{r}, \mathbf{v})$ as a solution to the linearized Boltzmann's equation

$$
(v-\mathrm{i} \omega) f_{1}(\mathbf{r}, \mathbf{v})+\mathbf{v} \frac{\partial f_{1}(\mathbf{r}, \mathbf{v})}{\partial \mathbf{r}}+e \mathbf{E}_{\mathrm{in}} \mathbf{v} \frac{\partial f_{0}(\varepsilon)}{\partial \varepsilon}=0,
$$

where $e$ is the electron charge. In equation (2.5) we have assumed that the collision integral

$$
\left(\partial f_{1} / \partial t\right)_{\mathrm{col}}=-f_{1} / \tau
$$

is evaluated in the relaxation time approximation ( $\tau=1 / v, v$ refers to electron collision frequency). Strictly speaking, $v \equiv v(T)$ is a temperature dependent value which can be presented as follows:

$$
v(T)=\frac{n_{\mathrm{e}} e^{2}}{m} \frac{K}{\Theta}\left(\frac{T}{\Theta}\right)^{5} \int_{0}^{\Theta / T} \frac{z^{5} e^{z}}{\left(e^{z}-1\right)^{2}} \mathrm{~d} z,
$$

where $n_{\mathrm{e}}$ is the electron concentration, $\Theta$ is the Debye temperature, and $K$ combines together the factors depending on the details of the Fermi surface geometry and scattering matrix elements.

For simplicity, we also assume that the vortex electric field induced by magnetic component of the external EM field gives a comparatively small input at the plasmon resonance frequencies and can be neglected in equation 2.5 .

What is more, the function $f_{1}(\mathbf{r}, \mathbf{v})$ ought to satisfy the boundary conditions as well. These conditions may be chosen from the character of electron reflection from the inner walls of the MN. We adopt, as is usually done, the assumption of diffusive electron scattering by the boundary of MN. Then, the boundary conditions can be presented in the form

$$
\left.f(\mathbf{r}, \mathbf{v})\right|_{S}=0, \quad \mathbf{v}_{\mathrm{n}}<0,
$$

where $\mathbf{v}_{\mathrm{n}}$ is the velocity of the component normal to the particle surface.

Along with diffusive scattering, the mirror boundary conditions at the nanoparticle surface were examined in the literature for electron scattering (see, e.g., [43, 44]). In this case, each electron is reflected from the surface at the same angle at which it falls to the surface. In diffuse reflection, the electron is reflected from the surface at any angle. For the mirror mechanism to be dominant, the surface must be perfectly smooth in the atomic scale because the degree of reflectivity of the boundary essentially depends on its smoothness. Practically, for a nonplanar border such smoothness is extremely difficult to achieve. As was shown [45], the mirror boundary conditions give a small correction to the results obtained with the account of only the diffusive electron reflections. Therefore, we choose more realistic boundary conditions given by equation (2.7).

The boundary conditions (2.7) in the case of an ellipsoidal MN, broadly speaking, depend on the angles, which complicates the solution of equation (2.5). 
It is rather easy to solve (2.5) and to satisfy the boundary conditions of 2.7) if one passes to a transformed coordinate system, where an ellipsoid with semiaxes $a, b, c$ (along the $x, y$, and $z$ directions, respectively) transforms into a sphere of radius $R$ with the same volume:

$$
x_{j}=\frac{d_{j}}{R} x_{j}^{\prime}, \quad R=(a b c)^{1 / 3},
$$

with $j=1,2,3$, and $d_{1}=a, d_{2}=b, d_{3}=c$. A similar transformation should be made for the electron velocities:

$$
v_{j}=\frac{d_{j}}{R} v_{j}^{\prime}
$$

Equation (2.5) and the boundary conditions 2.7) in transformed coordinate and velocity systems can be rewritten as follows:

$$
\begin{gathered}
(v-\mathrm{i} \omega) f_{1}\left(\mathbf{r}^{\prime}, \mathbf{v}^{\prime}\right)+\mathbf{v}^{\prime} \frac{\partial f_{1}\left(\mathbf{r}^{\prime}, \mathbf{v}^{\prime}\right)}{\partial \mathbf{r}^{\prime}}+e \mathbf{E}_{\text {in }} \mathbf{v}^{\prime} \frac{\partial f_{0}(\varepsilon)}{\partial \varepsilon}=0, \\
\left.f\left(\mathbf{r}^{\prime}, \mathbf{v}^{\prime}\right)\right|_{r^{\prime}=R}=0, \quad \mathbf{r}^{\prime} \cdot \mathbf{v}^{\prime}<0 .
\end{gathered}
$$

The first condition calls for zero equality of the electron distribution function at the nanoparticle surface, and the second one, $\mathbf{r}^{\prime} \cdot \mathbf{v}^{\prime}<0$, means that an electron motion occurs only inside MN, and there is no electron leakage through the NP surface.

Equation (2.10) presents the partial differential equation with boundary conditions (2.11). Using the method of characteristics, one can obtain the solution to this equation in the form

$$
f_{1}\left(\mathbf{r}^{\prime}, \mathbf{v}^{\prime}, t\right)=-e \frac{\partial f_{0}}{\partial \varepsilon} \mathbf{v}^{\prime} \mathbf{E}_{\mathrm{in}} \frac{1-\exp \left[-(v-\mathrm{i} \omega) t_{\mathrm{c}}\left(\mathbf{r}^{\prime}, \mathbf{v}^{\prime}\right)\right]}{v-\mathrm{i} \omega},
$$

where the characteristic $t_{\mathrm{c}}\left(\mathbf{r}^{\prime}, \mathbf{v}^{\prime}\right)$ can be presented as follows:

$$
t_{\mathrm{c}}\left(\mathbf{r}^{\prime}, \mathbf{v}^{\prime}\right)=\frac{1}{\mathbf{v}^{\prime 2}}\left[\mathbf{r}^{\prime} \mathbf{v}^{\prime}+\sqrt{\left(R^{2}-r^{\prime 2}\right) \mathbf{v}^{\prime 2}+\left(\mathbf{r}^{\prime} \mathbf{v}^{\prime}\right)^{2}}\right] .
$$

The radius vector $\mathbf{R}$ determines the starting position of an electron at the moment $t_{\mathrm{c}}=0$. The characteristic curve of equation (2.13) depends only on the absolute value of $\mathbf{R}$ and does not depend on the direction of $\mathbf{R}$.

It is reasonable to point out that, in spite of the electric field which remains spatially uniform inside the MN, the distribution function (2.12) still depends on the coordinates due to the requirement to obey the boundary conditions (2.11). Owing to this dependence, other physical parameters averaged with $f_{1}\left(\mathbf{r}^{\prime}, \mathbf{v}^{\prime}\right)$ start to depend on coordinates too. Since the physical sensing involves only parameters averaged over the whole MN volume $V$, it is necessary to fulfill the integration over all the coordinates inside the MN. One can see this exemplified just below.

Using the solution (2.12), one can calculate the density of a high-frequency current induced by the EM wave inside the MN. Performing the Fourier transformation of equation (2.12), we obtain

$$
\mathbf{j}(\omega)=\frac{2 e}{V}\left(\frac{m}{2 \pi \hbar}\right)^{3} \iiint_{V} \mathrm{~d}^{3} r^{\prime} \iiint \mathrm{d}^{3} v^{\prime} \mathbf{v}^{\prime} f_{1}\left(\mathbf{r}^{\prime}, \mathbf{v}^{\prime}, \omega\right) .
$$

\section{Electric-conductivity tensor}

Let us introduce the tensor of electric conductivity $\sigma_{\alpha \beta}(\omega)$ using the relationship

$$
j_{\alpha}(\omega)=\sum_{\beta=1}^{3} \sigma_{\alpha \beta}(\omega) E_{\text {in }}^{(\beta)}(\omega)
$$

Then, in accordance with both equations (2.12) and (2.14), the components of this tensor can be presented as follows:

$$
\sigma_{\alpha \beta}(\omega)=-\frac{2 e}{V}\left(\frac{m}{2 \pi \hbar}\right)^{3} \iiint_{V} \mathrm{~d}^{3} r^{\prime} \iiint \mathrm{d}^{3} v^{\prime} v_{\alpha}\left[e v_{\beta} \frac{\partial f_{0}}{\partial \varepsilon} \frac{1-\mathrm{e}^{-(v-\mathrm{i} \omega) t_{\mathrm{c}}\left(r^{\prime}, v^{\prime}\right)}}{v-\mathrm{i} \omega}\right] .
$$


Electric-conductivity tensor $\sigma_{\alpha \beta}=\sigma_{\beta \alpha}$ is the symmetrical second-rank tensor. Since the integrand in equation (3.2) is an odd function for nondiagonal tensor components $\alpha \neq \beta$, and the integration is conducted over the whole velocity space $[-\infty, \infty]$, only diagonal components in this equation have been retained.

If we introduce a rectangular coordinate system and choose the fundamental directions $x, y, z$ to be coincident with the three principal axes of the ellipsoids, then we get

$$
\sigma=\left(\begin{array}{ccc}
\sigma_{x x} & 0 & 0 \\
0 & \sigma_{y y} & 0 \\
0 & 0 & \sigma_{z z}
\end{array}\right)
$$

The tensor of electric conductivity, as can be seen from equation (3.2), is a complex value:

$$
\sigma_{\alpha \beta}(\omega)=\sigma_{\alpha \beta}^{\prime}(\omega)+\mathrm{i} \sigma_{\alpha \beta}^{\prime \prime}(\omega) .
$$

The surface effect on the conducting phenomenon is described in equation (3.2) by means of a characteristic $t_{\mathrm{c}}\left(\mathbf{r}^{\prime}, \mathbf{v}^{\prime}\right)$. It accounts for the restrictions imposed on the electron movement by nanoparticle surfaces. As one can see from equation (2.12), the value of $t_{\mathrm{c}}$ is of the order of $t^{\prime} \sim R / v_{\mathrm{F}}$, where $v_{\mathrm{F}}$ is the Fermi velocity. This implies that the value reciprocal to $t_{\mathrm{c}}$ will correspond to the vibration frequency between the particle walls. Hence, the inequality $v t_{\mathrm{c}} \gg 1$ indicates that the electron collision frequency inside the MN bulk would significantly exceed the one for an electron collision with the surface of MN. If this inequality is satisfied, one can direct $t_{\mathrm{c}} \rightarrow \infty$. Then, the exponent in equation (3.2) vanishes and finally we arrive at a standard expression for an electric-conductivity [1, 42]

$$
\sigma(\omega)=\frac{1}{4 \pi} \frac{\omega_{\mathrm{pl}}^{2}}{v-\mathrm{i} \omega},
$$

with

$$
\omega_{\mathrm{pl}}^{2}=\frac{4 e^{2} m^{2} v_{\mathrm{F}}^{3}}{3 \pi \hbar^{3}}
$$

To be sure of that, it is necessary to take into account that the energy derivative of $f_{0}$ in the zero approximation in the small ratio of $k_{\mathrm{B}} T / \varepsilon_{\mathrm{F}}\left(\varepsilon_{\mathrm{F}}\right.$ is the Fermi energy) can be replaced by

$$
\frac{\partial f_{0}}{\partial \varepsilon} \approx-\delta\left(\varepsilon-\varepsilon_{\mathrm{F}}\right)
$$

and passes in equation (3.2) to the integration over $v$ in the spherical coordinate system

$$
\iint_{-\infty}^{\infty} \int_{0}^{3} \mathrm{~d}^{3} v \rightarrow \int_{0}^{2 \pi} \mathrm{d} \varphi \int_{0}^{\pi} \sin \theta \mathrm{d} \theta \int_{0}^{\infty} v^{2} \mathrm{~d} v,
$$

with the use of the formula

$$
\int_{0}^{\infty} v^{4} \delta\left(v^{2}-v_{\mathrm{F}}^{2}\right) \mathrm{d} v=\frac{v_{\mathrm{F}}^{3}}{2} .
$$

We denote with $\varphi$ and $\theta$ the azimuthal and polar angles with respect to the ellipsoid rotation axis $z$, respectively. Note here that only diagonal terms with $v_{\alpha}=v_{\beta}=v$ are retained after integration over all angles. As one can see from equation (3.4), the conductivity becomes a scalar quantity in this approximation.

In the general case of an ellipsoidal-shaped MN, the electric conductivity is the tensor quantity (3.2). Integrating it over all nanoparticle coordinates in a solid angle $\mathrm{d} \Omega=\sin \theta \mathrm{d} \varphi \mathrm{d} \theta$ gives

$$
\frac{1}{V} \iiint_{V} \mathrm{~d}^{3} r^{\prime}\left[1-\mathrm{e}^{-(v-\mathrm{i} \omega) t^{\prime}\left(\mathbf{r}^{\prime}, v^{\prime}\right)}\right]=\frac{3}{4} \Psi\left(\omega, v^{\prime}\right),
$$

where we use 


$$
\int_{0}^{\infty} \mathrm{d} z z^{3} \operatorname{Erfc}\left(z+\frac{a}{z}\right)=\frac{1}{4}\left(a+\frac{3}{4}\right) \mathrm{e}^{-4 a}
$$

provided that $\operatorname{Re} a>0$, and

$$
\int_{0}^{\infty} \mathrm{d} z z^{3} \operatorname{Erfc}\left(z-\frac{a}{z}\right)=\frac{1}{2}\left(a^{2}+a+\frac{3}{8}\right) .
$$

The complex $\Psi$ function entering the equation (3.8) has the form

$$
\Psi\left(\omega, v^{\prime}\right)=\Phi\left(\omega, v^{\prime}\right)-\frac{4}{q^{2}}\left(1+\frac{1}{q}\right) \mathrm{e}^{-q},
$$

with

$$
\Phi\left(\omega, v^{\prime}\right)=\frac{4}{3}-\frac{2}{q}+\frac{4}{q^{3}}, \quad q \equiv q\left(\omega, v^{\prime}\right)=\frac{2 R}{v^{\prime}}(v-\mathrm{i} \omega),
$$

and $v^{\prime}(=\varsigma v)$ is a "deformed" electron velocity [17] with the coefficient of "deformation" $\varsigma_{j}=R / d_{j}$. The last summand in equation (3.11) represents the oscillation part of the $\Psi$ function and the first one refers to its smooth part.

Accounting for equation (3.8) and only diagonal components in equation (3.2), there remain the integrals over all electron velocities. To calculate them, we pass to a spherical coordinate system with the $z$ axis directed along the rotation axis of the spheroid (as we have done it above). Then, equation (3.2) can be rewritten as follows:

$$
\sigma_{j j}(\omega)=\frac{3 e^{2} m^{3}}{2(2 \pi \hbar)^{3}} \frac{1}{v-\mathrm{i} \omega} \int_{0}^{2 \pi} \mathrm{d} \varphi \int_{0}^{\pi} \sin \theta \mathrm{d} \theta \int_{0}^{\infty} v^{2} \mathrm{~d} v v_{j}^{2}\left(-\frac{\partial f_{0}}{\partial \varepsilon}\right) \Psi\left(\omega, v^{\prime}\right),
$$

where $v_{j}$ is the $j$-th component of the electron velocity, $j=x, y, z$, with

$$
\left\{\begin{array}{l}
v_{\left(\begin{array}{l}
x \\
y
\end{array}\right)}^{2}=v^{2} \sin ^{2} \theta \cdot\left(\begin{array}{c}
\cos ^{2} \varphi \\
\sin ^{2} \varphi
\end{array}\right) \\
v_{z}^{2}=v^{2} \cos ^{2} \theta
\end{array}\right.
$$

respectively.

It should be noted that the "deformed" electron velocity entering the $\Psi$ function can be expressed through the electron velocity in a Cartesian coordinate system as follows:

$$
v^{\prime}=v R \sqrt{\left(\frac{\cos ^{2} \varphi}{a^{2}}+\frac{\sin ^{2} \varphi}{b^{2}}\right) \sin ^{2} \theta+\frac{\cos ^{2} \theta}{c^{2}}}
$$

Let us suppose that the particle in the matrix is modelled as a rotationally symmetric ellipsoid ( $a=$ $\left.b \equiv R_{\perp}, c \equiv R_{\|}\right)$with the symmetry axis along the $z$ direction. The components of an electron velocity parallel $\left(v_{\|}\right)$and perpendicular $\left(v_{\perp}\right)$ to the spheroid revolution axis

$$
v_{\|}=v_{z}=v \cos \theta, \quad v_{\perp}=\sqrt{v_{x}^{2}+v_{y}^{2}}=v \sin \theta
$$

play an important role in this case, and the $v^{\prime}$ ceases to depend on the angle $\varphi$.

Let us pass from an integration in equation (3.13) over electron velocities to the integration over electron energies

$$
v^{4} \mathrm{~d} v=\frac{1}{m}\left(\frac{2 \varepsilon}{m}\right)^{3 / 2} \mathrm{~d} \varepsilon
$$

and take into account the integrals

$$
\int_{0}^{2 \pi} \cos ^{2} \varphi \mathrm{d} \varphi=\int_{0}^{2 \pi} \sin ^{2} \varphi \mathrm{d} \varphi=\pi
$$


Then, with the use of equations (3.13), 3.16), and (3.17), we obtain for the main components of the complex electric conductivity tensor, namely for

$$
\sigma_{x x}=\sigma_{y y} \equiv \sigma_{\perp}, \quad \sigma_{z z}=\sigma_{\|},
$$

the expression

$$
\sigma_{\left(\begin{array}{l}
\| \\
\perp
\end{array}\right)}(\omega)=\frac{3 e^{2}}{2 \pi^{2}} \frac{\sqrt{2 m}}{\hbar^{3}}\left[\frac{1}{v-\mathrm{i} \omega} \int_{0}^{\pi / 2}\left(\begin{array}{c}
\cos \theta^{\prime} \sin ^{2} \theta^{\prime} \\
\frac{1}{2} \cos ^{3} \theta^{\prime}
\end{array}\right) \mathrm{d} \theta^{\prime} \int_{0}^{\infty} \Psi\left(\omega, \varepsilon_{\theta^{\prime}}^{\prime}\right)\left(-\frac{\partial f_{0}}{\partial \varepsilon}\right) \varepsilon^{3 / 2} \mathrm{~d} \varepsilon\right] .
$$

We denote with $\theta^{\prime}(=\theta-\pi / 2)$ the angle between the direction of an electron velocity and an axis perpendicular to the spheroid rotation axes. Here, and below, the upper (lower) symbol in the parentheses on the left-hand side of equation (3.19) corresponds to the upper (lower) expression in the parentheses on the right-hand side of this equation.

The $\Psi$ function in (3.19) depends now on both the energy $\varepsilon^{\prime}$ and the angle $\theta^{\prime}$ because the parameter $q$ [see (3.12) and (3.14)] for a spheroidal particle becomes dependent on the angle $\theta$ and can be determined as

$$
q=\sqrt{\frac{2 m}{\varepsilon}}(v-\mathrm{i} \omega)\left(\frac{\sin ^{2} \theta}{R_{\perp}^{2}}+\frac{\cos ^{2} \theta}{R_{\|}^{2}}\right)^{-1 / 2} \equiv q(\theta, \varepsilon),
$$

where $R_{\|}$and $R_{\perp}$ are the semiaxes of the spheroid. The electron energy $\varepsilon^{\prime}$ entering the $\Psi$ function, becomes dependent on the angle $\theta$ too, and for a spheroid can be presented as follows:

$$
\varepsilon_{\theta^{\prime}}^{\prime}=\varepsilon R^{2}\left(\frac{\cos ^{2} \theta^{\prime}}{R_{\perp}^{2}}+\frac{\sin ^{2} \theta^{\prime}}{R_{\|}^{2}}\right),
$$

where the equation (3.14) was used. Since the spheroid semiaxes $R_{\perp}$ and $R_{\|}$can be easily expressed through the radius of a sphere $R$ of an equivalent volume

$$
R_{\perp}=R\left(\frac{R_{\perp}}{R_{\|}}\right)^{1 / 3}, \quad R_{\|}=R\left(\frac{R_{\perp}}{R_{\|}}\right)^{-2 / 3},
$$

the energy $\varepsilon^{\prime}$ does not depend on the particle radius $R$ but depends only on the spheroid axes ratio.

If we restrict ourselves here to the case of low temperatures, then the equation (3.6) can be used, and we find

$$
\int_{0}^{\infty} \Psi\left(\omega, \varepsilon_{\theta^{\prime}}^{\prime}\right) \delta\left(\varepsilon-\varepsilon_{\mathrm{F}}\right) \varepsilon^{3 / 2} \mathrm{~d} \varepsilon=\mu_{0}^{3 / 2} \Psi\left(\omega, \mu_{\theta^{\prime}}^{\prime}\right),
$$

where $\mu_{0}$ is the chemical potential at zero temperature. Substituting (3.23) into equation (3.19) and accounting that the electron concentration at $T=0$ can be presented as

$$
n_{0}=\frac{\left(2 m \mu_{0}\right)^{3 / 2}}{3 \pi^{2} \hbar^{3}}
$$

it is easy to check that equation [3.19) transforms to the form known from (see, e.g., [46]).

\subsection{Conductivity of a spherical MN}

For particles having a spherical shape, the electric conductivity becomes a scalar quantity, and one can put $R_{\|}=R_{\perp} \equiv R, \varepsilon^{\prime}=\varepsilon$ in equations (3.20) and (3.16); then, $q$ and the $\Psi$ function cease to dependent on the angle $\theta^{\prime}$,

$$
\int_{0}^{\pi / 2}\left(\begin{array}{c}
\cos \theta^{\prime} \sin ^{2} \theta^{\prime} \\
\frac{1}{2} \cos ^{3} \theta^{\prime}
\end{array}\right) \mathrm{d} \theta^{\prime}=\frac{1}{3}
$$


and equation (3.19) reduces to the form

$$
\sigma_{\mathrm{sph}}(\omega)=\frac{e^{2} \sqrt{2 m}}{2 \pi^{2} \hbar^{3}(v-\mathrm{i} \omega)} \int_{0}^{\infty} \Psi(\omega, \varepsilon)\left(-\frac{\partial f_{0}}{\partial \varepsilon}\right) \varepsilon^{3 / 2} \mathrm{~d} \varepsilon .
$$

Let us make the following change of variables in equation (3.26)

$$
\eta=\frac{\varepsilon-\mu}{k_{\mathrm{B}} T}, \quad \mathrm{~d} \eta=\frac{\mathrm{d} \varepsilon}{k_{\mathrm{B}} T} .
$$

Then

$$
-\frac{\partial f_{0}}{\partial \varepsilon} \mathrm{d} \varepsilon=\frac{\mathrm{e}^{-\eta}}{\left(1+\mathrm{e}^{-\eta}\right)^{2}} \mathrm{~d} \eta
$$

and the integral in (3.26) can be presented as

$$
\int_{-\mu /\left(k_{\mathrm{B}} T\right)}^{\infty} \Psi(\omega, \eta)\left(\mu+\eta k_{\mathrm{B}} T\right)^{3 / 2} \frac{\mathrm{e}^{-\eta}}{\left(1+\mathrm{e}^{-\eta}\right)^{2}} \mathrm{~d} \eta \equiv I,
$$

where

$$
\begin{aligned}
\Psi(\omega, \eta)= & \frac{4}{3}-\frac{\sqrt{\frac{2}{m}(\mu+\eta k T)}}{R(v-\mathrm{i} \omega)}+\frac{\left[\frac{2}{m}(\mu+\eta k T)\right]^{3 / 2}}{2 R^{3}(v-\mathrm{i} \omega)^{3}} \\
& -\frac{\frac{2}{m}(\mu+\eta k T)}{R^{2}(v-\mathrm{i} \omega)^{2}}\left[1+\frac{\sqrt{\frac{2}{m}(\mu+\eta k T)}}{2 R(v-\mathrm{i} \omega)}\right] \exp \left[-\frac{2 R(v-i \omega)}{\sqrt{\frac{2}{m}(\mu+\eta k T)}}\right],
\end{aligned}
$$

with [47]

$$
\mu \equiv \mu(T) \simeq \mu_{0}\left[1-\frac{\pi^{2}}{12}\left(\frac{k_{\mathrm{B}} T}{\mu_{0}}\right)^{2}\right] .
$$

Since the input of the integrand tends to zero as $\eta \rightarrow-\infty$, the lower limit in this integral can be extended to the $-\infty$. The derivative $\left(-\partial f_{0} / \partial \varepsilon\right.$ ) has a maximum at the point $\varepsilon=\mu$. So, it is conveniently to expand the product of $\Psi \cdot\left(\mu+\eta k_{\mathrm{B}} T\right)^{3 / 2} \equiv \Xi(\eta)$ in the powers of $\eta$

$$
\begin{aligned}
\Xi(\eta) \simeq & \mu^{3 / 2} \Psi(0)+\left[\frac{3}{2} k_{\mathrm{B}} T \sqrt{\mu} \Psi(0)+\mu^{3 / 2} \Psi^{\prime}(0)\right] \eta \\
& +\frac{1}{8 \sqrt{\mu}}\left[3\left(k_{\mathrm{B}} T\right)^{2} \Psi(0)+12 k_{\mathrm{B}} T \mu \Psi^{\prime}(0)+4 \mu^{2} \Psi^{\prime \prime}(0)\right] \eta^{2}+0[\eta]^{3},
\end{aligned}
$$

with

$$
\Psi(0)=\left.\Psi(\omega, \eta)\right|_{\eta \rightarrow 0}, \quad \Psi^{\prime}(0)=\left.\frac{\partial}{\partial \eta} \Psi(\omega, \eta)\right|_{\eta \rightarrow 0}, \quad \Psi^{\prime \prime}(0)=\left.\frac{\partial^{2}}{\partial \eta^{2}} \Psi(\omega, \eta)\right|_{\eta \rightarrow 0} .
$$

Note, that $\Psi(0)$ at $v^{\prime}=v_{\mathrm{F}}$ coincides with the $\Psi(\omega)$ defined by equation (3.11).

Then, the integral (3.29) can be presented as the sum of integrals

$$
\begin{aligned}
I \simeq \quad & \mu^{3 / 2} \Psi(0) I_{1}+\left[\frac{3}{2} k_{\mathrm{B}} T \sqrt{\mu} \Psi(0)+\mu^{3 / 2} \Psi^{\prime}(0)\right] I_{2} \\
& +\frac{1}{2}\left[\frac{3\left(k_{\mathrm{B}} T\right)^{2}}{4 \sqrt{\mu}} \Psi(0)+3 k_{\mathrm{B}} T \sqrt{\mu} \Psi^{\prime}(0)+\mu^{3 / 2} \Psi^{\prime \prime}(0)\right] I_{3},
\end{aligned}
$$

where the integrals $I_{j}=\int_{-\infty}^{\infty} \mathrm{d} \eta \eta^{j-1} \exp (-\eta) /[1+\exp (-\eta)]^{2}$ are calculated to be: $I_{1}=1, I_{2}=0$ and $I_{3}=$ $\pi^{2} / 6$. 
Using $I_{j}$ and (3.27, equation (3.26) transforms into

$$
\sigma_{\mathrm{sph}}(\omega)=\frac{e^{2} \sqrt{2 m \mu}}{2 \pi^{2} \hbar^{3}(v-\mathrm{i} \omega)}\left\{\mu\left[1+\frac{\pi^{2}}{8}\left(\frac{k_{\mathrm{B}} T}{\mu}\right)^{2}\right] \Psi(0)+\frac{\pi^{2}}{6}\left[3 k_{\mathrm{B}} T \Psi^{\prime}(0)+\mu \Psi^{\prime \prime}(0)\right]+0[\eta]^{3}\right\} .
$$

Here,

$$
\begin{aligned}
\Psi^{\prime}(0) & =\frac{k_{\mathrm{B}} T}{\mu q_{\mu}}\left[-1+\frac{6}{q_{\mu}^{2}}-\frac{6}{q_{\mu}} \mathrm{e}^{-q_{\mu}}\left(1+\frac{1}{q_{\mu}}+\frac{q_{\mu}}{3}\right)\right], \\
\Psi^{\prime \prime}(0) & =\frac{\left(k_{\mathrm{B}} T\right)^{2}}{2 \mu^{2} q_{\mu}}\left[1+\frac{6}{q_{\mu}^{2}}-4 \mathrm{e}^{-q_{\mu}}\left(1+\frac{q_{\mu}}{2}+\frac{3}{2 q_{\mu}}+\frac{3}{2 q_{\mu}^{2}}\right)\right],
\end{aligned}
$$

with

$$
q_{\mu}=\frac{2 R(v-\mathrm{i} \omega)}{\sqrt{2 \mu / m}} .
$$

Substituting (3.36)-3.37) into equation (3.35), we obtain

$$
\sigma_{\mathrm{sph}}(\omega, T)=\frac{m e^{2} \mu R}{\pi^{2} \hbar^{3} q_{\mu}}\left[\Psi(0)+\alpha_{T} \Psi_{T}(\omega)\right],
$$

with

$$
\begin{gathered}
\alpha_{T}=\frac{\pi^{2}}{6}\left(\frac{k_{\mathrm{B}} T}{\mu}\right)^{2}, \\
\Psi_{T}(\omega)=\Phi_{T}(\omega)-\left[1+\frac{8}{q_{\mu}}+\frac{24}{q_{\mu}^{2}}\left(1+\frac{1}{q_{\mu}}\right)\right] \mathrm{e}^{-q_{\mu}}, \\
\Phi_{T}(\omega)=1-\frac{4}{q_{\mu}}+\frac{24}{q_{\mu}^{3}} .
\end{gathered}
$$

When the temperature $T \rightarrow 0$ in equation (3.40), then (3.39) is reduced to the form

$$
\sigma_{\mathrm{sph}}(\omega)=\frac{e^{2} \mu_{0}^{3 / 2} \sqrt{2 m}}{2 \pi^{2} \hbar^{3}} \frac{\Psi(0)}{v-\mathrm{i} \omega}
$$

known from other calculations (see, e.g., [48]).

The conductivity of MN given by (3.39) is a complex value. Practically, we need to know both the real and the imaginary parts of it.

Further analytical calculations are possible for some particular cases. In the case of a spherical MN, there are three actual frequencies that are usually considered: the frequency of an incident electromagnetic field $\omega$, the collision frequency of electrons in the particle volume $v$, and the vibration frequency between the particle walls $v_{\mathrm{S}}=v_{\mathrm{F}} /(2 R)$ (provided the particle size is less than the electron mean free path). When $v>v_{\mathrm{s}}$, the mechanism of an electron scattering in the bulk dominates, and an electron scattering from the particle surface gives only small corrections of the order of $v_{\mathrm{s}} / v$. However, a much more interesting case is when the mechanism of the surface electron scattering dominates, which corresponds to the inequality $v<v_{\mathrm{s}}$.

Let us suppose that the mean free path of a conduction electron $l$ is much greater than the particle size. In this case, an electron scattering occurs mainly from the inner surface of the MN. The electrons oscillate between the walls of the particle with the frequency $v_{\mathrm{s}}$. In the case of $v \ll v_{\mathrm{s}}$, to a first approximation, one can formally put $v \rightarrow 0$. If one introduces the designation

$$
q_{i}=\frac{\omega}{v_{\mathrm{s}}}
$$

then for real and imaginary parts of the ratios $\Psi /(v-\mathrm{i} \omega)$ and $\Psi_{T} /(v-\mathrm{i} \omega)$ we obtain

$$
\begin{aligned}
& \operatorname{Re}\left[\frac{\Psi}{v-\mathrm{i} \omega}\right]_{v \rightarrow 0}=\frac{1}{\omega}\left[\frac{2}{q_{i}}-\frac{4}{q_{i}^{2}} \sin q_{i}+\frac{4}{q_{i}^{3}}\left(1-\cos q_{i}\right)\right], \\
& \operatorname{Im}\left[\frac{\Psi}{v-\mathrm{i} \omega}\right]_{v \rightarrow 0}=\frac{4}{\omega}\left[\frac{1}{3}+\frac{1}{q_{i}^{2}}\left(\cos q_{i}-\frac{\sin q_{i}}{q_{i}}\right)\right],
\end{aligned}
$$


and

$$
\begin{aligned}
& \operatorname{Re}\left[\frac{\Psi_{T}}{v-\mathrm{i} \omega}\right]_{v \rightarrow 0}=\frac{1}{\omega}\left[\left(1-\frac{24}{q_{i}^{2}}\right) \sin q_{i}+\frac{4}{q_{i}}\left(1+\frac{6}{q_{i}^{2}}\right)+\frac{8}{q_{i}}\left(1-\frac{3}{q_{i}^{2}}\right) \cos q_{i}\right], \\
& \operatorname{Im}\left[\frac{\Psi_{T}}{v-\mathrm{i} \omega}\right]_{v \rightarrow 0}=\frac{1}{\omega}\left[1-\left(1-\frac{24}{q_{i}^{2}}\right) \cos q_{i}+\frac{8}{q_{i}}\left(1-\frac{3}{q_{i}^{2}}\right) \sin q_{i}\right] .
\end{aligned}
$$

Substituting (3.45)-(3.48) into equation (3.39), one obtains for the real and imaginary parts of $\sigma$ the following expressions:

$$
\begin{aligned}
\sigma_{\mathrm{sph}}^{\prime}(\omega, T)= & \frac{3}{4} \frac{e^{2} n_{\mathrm{e}}(T)}{m \omega}\left\{\frac{2}{q_{i}}-\frac{4}{q_{i}^{2}} \sin q_{i}+\frac{4}{q_{i}^{3}}\left(1-\cos q_{i}\right)\right. \\
& \left.+\alpha_{T}\left[\frac{4}{q_{i}}+\frac{24}{q_{i}^{3}}+\left(1-\frac{24}{q_{i}^{2}}\right) \sin q_{i}+\frac{8}{q_{i}}\left(1-\frac{3}{q_{i}^{2}}\right) \cos q_{i}\right]\right\}, \\
\sigma_{\mathrm{sph}}^{\prime \prime}(\omega, T)= & \frac{3}{4} \frac{e^{2} n_{\mathrm{e}}(T)}{m \omega}\left\{\frac{4}{3}+\frac{4}{q_{i}^{2}}\left(\cos q_{i}-\frac{\sin q_{i}}{q_{i}}\right)\right. \\
& \left.+\alpha_{T}\left[1+\frac{8}{q_{i}}\left(1-\frac{3}{q_{i}^{2}}\right) \sin q_{i}-\left(1-\frac{24}{q_{i}^{2}}\right) \cos q_{i}\right]\right\}
\end{aligned}
$$

provided that $v \ll v_{\mathrm{s}}$. In the presented equations, the electron concentration depends on the temperature as [47]

$$
n_{\mathrm{e}}(T)=n_{0}\left\{1+\frac{\pi^{2}}{8}\left[\frac{k_{\mathrm{B}} T}{\mu}\right]^{2}\right\} .
$$

If one neglects the oscillation terms in the equation (3.50), then we obtain

$$
\sigma_{\mathrm{sph}}^{\prime \prime}(\omega, T) \simeq \frac{\omega_{\mathrm{pl}}^{2}(T)}{4 \pi \omega}\left(1+\frac{3}{4} \alpha_{T}\right),
$$

with $\omega_{\mathrm{pl}}^{2}(T)=4 \pi e^{2} n_{\mathrm{e}}(T) / m$.

At $T \rightarrow 0$, equations (3.49) and (3.50) agree with the expressions known from earlier calculations [46, 48].

\subsection{Linewidth and "figure of merit"}

The linewidth of the particle-plasmon resonance is controlled by lifetime broadening due to various decay processes. Knowing the expressions for conductivity tensor (3.19) and (3.49), the halfwidth of the plasmon line absorption can be calculated simply by means of a relation

$$
\Gamma_{j}(\omega, T)=\frac{4 \pi}{\epsilon_{\infty}+n^{2}\left(1 / L_{j}-1\right)} \operatorname{Re} \sigma_{j j}(\omega, T),
$$

where $n\left(=\sqrt{\epsilon_{\mathrm{m}}}\right)$ is the refractive index of the surrounding medium and $\varepsilon_{\infty} \equiv 1+\epsilon_{\text {inter }}$ is the high frequency dielectric constant due to interband and core transitions of the inner electrons in a MN's material.

The "figure of merit" (FOM) can be expressed through the $\sigma_{j j}$ as well. For MNs having a spheroidal shape we found [48]

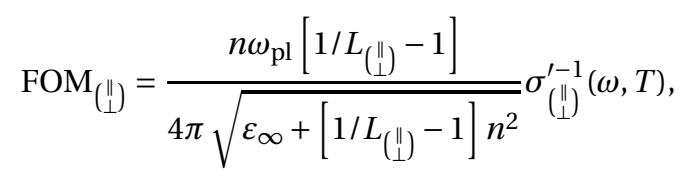

with geometrical factors [49]

$$
L_{\|}=\frac{1}{\left(1+R_{\|} / R_{\perp}\right)^{1.6}}, \quad L_{\perp}=\frac{1}{2}\left(1-L_{\|}\right) .
$$


Finally, it should be reminded that the real and imaginary parts of a dielectric constant tensor $\epsilon_{j j}$ can be easily obtained with the help of tensor $\sigma_{j j}$, as [47]

$$
\begin{aligned}
& \epsilon_{j j}^{\prime}(\omega, T)=\epsilon_{\infty}-\frac{4 \pi}{\omega} \sigma_{j j}^{\prime \prime}(\omega, T), \\
& \epsilon_{j j}^{\prime \prime}(\omega, T)=\frac{4 \pi}{\omega} \sigma_{j j}^{\prime}(\omega, T) .
\end{aligned}
$$

The sign in the last equation and before the second term in equation (3.56) corresponds to the exponent sign in time dependence of an external electric field $\mathbf{E}(t)=\mathbf{E}_{0} \mathrm{e}^{-\mathrm{i} \omega t}$. The imaginary part of the dielectric constant provides basically the damping and broadening of SPR.

The linewidth of SPR and FOM are strongly dependent on the particle radius. We will illustrate this dependence below, having built the temperature behavior of SPR linewidth for different $R$ as an example.

The SPR linewidth is plotted in figure 1 against temperature for spherical Au nanoparticles with two different radii embedded in the water $(n=1.33)$. Calculations were made according to the formulas (3.53) and [3.49) for the following parameters of Au nanoparticle [50]: $v_{\mathrm{F}}=1.394 \times 10^{8} \mathrm{~cm} / \mathrm{s}, T_{\mathrm{F}}=6.41 \times 10^{4} \mathrm{~K}$, $\omega_{\mathrm{pl}}=1.37 \times 10^{16} \mathrm{~s}^{-1}$, and $\epsilon_{\infty}=9.84$. The electron concentration was estimated from equation (3.51) with $n_{0}$ given above by equation (3.24). For $\mathrm{Au}, n_{0} \simeq 5.9 \times 10^{22} \mathrm{~cm}^{-3}$. The behavior of $\Gamma(T)$ is governed by the temperature dependence of the $\epsilon^{\prime \prime}(T)$ in accordance with equations (3.53) and (3.57).

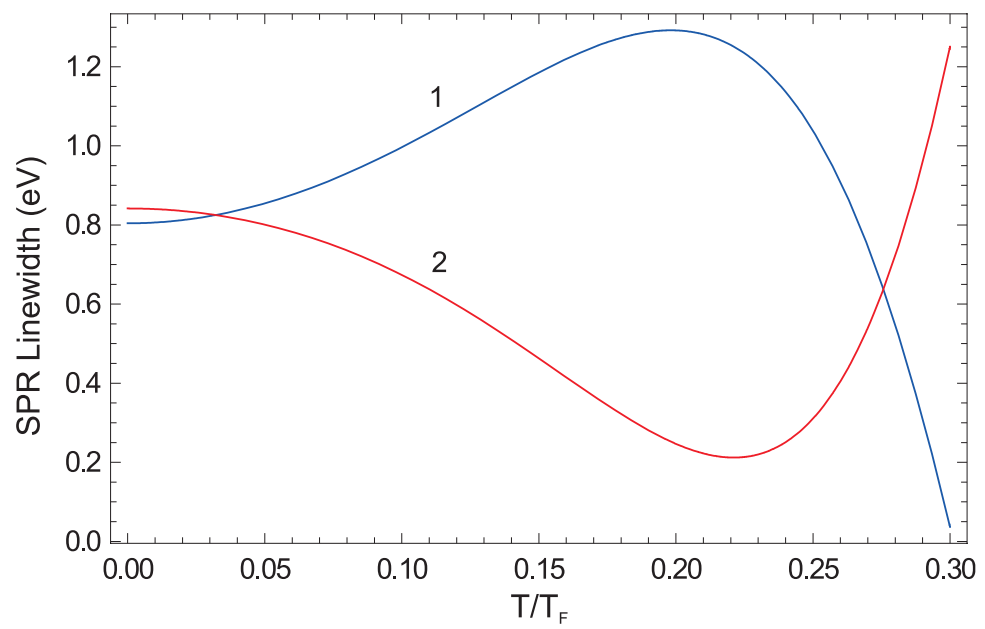

Figure 1. (Color online) SPR linewidth vs temperature for spherical Au particles with different $R(\AA): 50$ (1) and 54.7 (2), embedded in water.

High temperature of an electron gas can be achieved inside the MN by using laser pumping at SPR frequency when hot electrons are produced. As the temperature of an electron gas decreases after an initial excitation, the SPR linewidth can increase (see curve 1, figure 1) or decrease (curve 2) depending on the particle radius. Such different behavior is due to the linewidth oscillations with the particle radius changing [48]. Earlier [30, 33], the results for Ag and Au nanoparticles were reported where the linewidth is only reduced linearly with the temperature lowering over a range of crystal temperatures.

Let us calculate the polarizability tensor for MNs at finite temperatures.

\section{Polarizability tensor}

The dipole electric moment for a spherical particle embedded in the media with dielectric constant $\epsilon_{\mathrm{m}}$, can be written as follows:

$$
d_{j}(\omega)=\epsilon_{\mathrm{m}} \sum_{k=1}^{3} \alpha_{j k}(\omega) E_{k}(\omega)
$$

where $E_{k}$ is the $k$ component of an external electric field and $\alpha_{j k}$ is the polarizability tensor of the MN. The calculation of $\mathbf{d}$ is especially simple for the case when the particle sizes are small compared to some 
"wavelength" $\delta \sim c /(\sqrt{|\epsilon|} \omega)$, which corresponds to the frequency $\omega$ in the particle bulk. In this case, one can calculate the polarizability of MN using the formulae obtained for an external uniform statical field. In general terms, this tensor can be treated as being a complex value

$$
\alpha_{j k}(\omega)=\alpha_{j k}^{\prime}(\omega)+\mathrm{i} \alpha_{j k}^{\prime \prime}(\omega) \text {. }
$$

Tensor of electric conductivity was introduced by equation (3.1). The component of the inner electric field in equation (3.1) is linked to the component of an external electric filed in equation (4.1) by expression (2.3). Since the density of electric current is related to the dipole moment by a simple relation

$$
j(t)=\frac{1}{V} \frac{\partial}{\partial t} d(t)
$$

it is easy to establish the connection between polarizability and conductivity of MN. Performing the Fourier transformation of equation (4.3), we obtain

$$
j(\omega)=\frac{\mathrm{i} \omega}{V} d(\omega) .
$$

Then, using equations (4.1), 4.4), and (3.1), the polarizability tensor can be expressed through the conductivity tensor $\alpha_{j j}$ by means of

$$
\alpha_{j j}(\omega, T)=-\frac{V}{\epsilon_{\mathrm{m}} \omega} \frac{\mathrm{i} \sigma_{j j}(\omega, T)}{1+L_{j}\left[\epsilon_{j j}(\omega) / \epsilon_{\mathrm{m}}-1\right]} .
$$

In equation (4.5) for $\mathrm{MN}$ with a spheroidal shape, parameter $V$ represents the volume of spheroid (= $\left.\frac{4}{3} \pi R_{\perp}^{2} R_{\|}\right)$.

Using equations (3.56) and (3.57) for an isolated MN in host, we come to the Clausius-Mossotti dipole polarizability [1]

$$
\alpha_{j j}(\omega, T)=\frac{V}{4 \pi L_{j}} \frac{\epsilon_{j j}(\omega, T)-\epsilon_{\mathrm{m}}}{\epsilon_{j j}(\omega, T)+\left(1 / L_{j}-1\right) \epsilon_{\mathrm{m}}},
$$

where tensor $\epsilon_{j j}$ corresponds to a given frequency $\omega$, and in the case of frequencies close to the plasma oscillations of electrons in metal, it can be presented as follows:

$$
\epsilon_{j j}(\omega, T) \simeq \epsilon_{\infty}-\left(\frac{\omega_{\mathrm{pl}}}{\omega}\right)^{2}+\frac{4 \pi}{\omega} \mathrm{i} \sigma_{j j}(\omega, T) .
$$

Optical absorption generally measures the $\operatorname{Im} \epsilon(\omega, T)$.

\section{Light absorption and scattering}

In classical electrodynamics it is supposed that the light scattering results from the polarization of the scattered particle when it is illuminated with a beam of light. Since the spatially uniform field causes only a dipole polarization, the nanoparticle scatters the light like a vibration dipole [1]. In the case of particles of a spheroidal shape, the dipole moment of the MN can be presented in the form [51]

$$
\mathbf{d}=\alpha_{\perp} \epsilon_{m} \mathbf{E}+\left(\alpha_{\|}-\alpha_{\perp}\right) \epsilon_{m}(\mathbf{m E}) \mathbf{m}
$$

where $\mathbf{m}$ is the unit vector directed along the spheroid axis of revolution. Accounting for $\mathbf{m} \cdot \mathbf{E}=E \cos \phi$, where $\phi$ is the angle between the spheroid axis of revolution and the direction of the electric field $\mathbf{E}$, the equation (5.1) can be rewritten as follows:

$$
\mathbf{d}=\sqrt{\alpha_{\perp}^{2} \sin ^{2} \phi+\alpha_{\|}^{2} \cos ^{2} \phi} \epsilon_{m} \mathbf{E} .
$$

Using our forerunning formulae for $\mathbf{d}$ and $\alpha$, it is easy to calculate the temperature dependence of light absorption and scattering. The overall absorption and scattering cross-sections for a spheroidal MN 
are given by

$$
\begin{aligned}
C_{\mathrm{abs}} & =4 \pi \frac{\omega}{c} \sqrt{\epsilon_{\mathrm{m}}}\left[\left(\operatorname{Im} \alpha_{\|}\right) \cos ^{2} \phi+\left(\operatorname{Im} \alpha_{\perp}\right) \sin ^{2} \phi\right], \\
C_{\mathrm{sca}} & =\frac{8}{3} \pi\left(\frac{\omega}{c} \sqrt{\epsilon_{\mathrm{m}}}\right)^{4}\left(\left|\alpha_{\|}\right|^{2} \cos ^{2} \phi+\left|\alpha_{\perp}\right|^{2} \sin ^{2} \phi\right) .
\end{aligned}
$$

The square of diagonal components of the polarizability tensor can be presented in the Lorenzian form as follows:

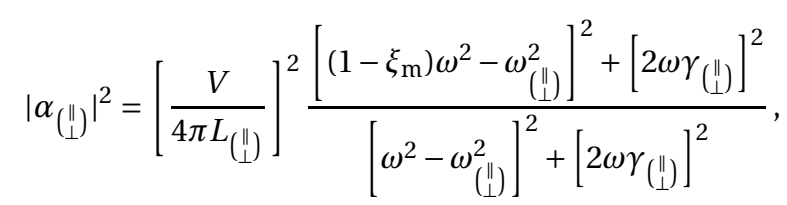

and the imaginary part of the polarizability tensor is

$$
\operatorname{Im} \alpha_{\left(\begin{array}{l}
\| \\
\perp
\end{array}\right)}=\left[\frac{V}{4 \pi L_{\left(\begin{array}{l}
\| \\
\perp
\end{array}\right)}}\right] \frac{2 \omega^{3} \xi_{\mathrm{m}} \gamma_{\left(\begin{array}{l}
\| \\
\perp
\end{array}\right)}}{\left[\omega^{2}-\omega_{\left(\begin{array}{l}
\| \\
\perp
\end{array}\right)}^{2}\right]^{2}+\left[2 \omega \gamma_{\left(\begin{array}{l}
\| \\
\perp
\end{array}\right)}\right]^{2}} .
$$

Here,

$$
\begin{gathered}
\xi_{\mathrm{m}}=\frac{\epsilon_{\mathrm{m}}}{\epsilon_{\mathrm{m}}+L_{\left(\begin{array}{l}
\| \\
\perp
\end{array}\right)}\left(1-\epsilon_{\mathrm{m}}\right)}, \\
\omega_{\left(\begin{array}{l}
\| \\
\perp
\end{array}\right)}^{2} \equiv \omega_{\left(\begin{array}{l}
\| \\
\perp
\end{array}\right)}^{2}(T)=\frac{L_{\left(\begin{array}{l}
\| \\
\perp
\end{array}\right)}}{\epsilon_{\mathrm{m}}+L_{\left(\begin{array}{l}
\| \\
\perp
\end{array}\right)}\left(1-\epsilon_{\mathrm{m}}\right)} \omega_{\mathrm{pl}}^{2}(T)
\end{gathered}
$$

are the $\|$ and $\perp$ surface plasmon frequencies, and

$$
\gamma_{\left(\begin{array}{l}
\| \\
\perp
\end{array}\right)} \equiv \gamma_{\left(\begin{array}{l}
\| \\
\perp
\end{array}\right)}(\omega, T)=\frac{2 \pi L_{\left(\begin{array}{l}
\| \\
\perp
\end{array}\right)}}{\epsilon_{\mathrm{m}}+L_{\left(\begin{array}{l}
\| \\
\perp
\end{array}\right)}\left(1-\epsilon_{\mathrm{m}}\right)} \operatorname{Re} \sigma_{\left(\begin{array}{l}
\| \\
\perp
\end{array}\right)}(\omega, T)
$$

is the half-width of the resonance for light polarized along $(\|)$ or across $(\perp)$ the rotation axis of the spheroid, $\sigma_{\|}, \sigma_{\perp}$ are the corresponding components of the conductivity tensor, and $L_{\|}, L_{\perp}$ are the geometrical factors given above by equations 3.55 .

On resonance, the polarizability tensor looks as follows:

$$
\alpha_{\left(\begin{array}{l}
\| \\
\perp
\end{array}\right)}(\omega, T) \simeq \frac{V}{8 \pi} \frac{\xi_{\mathrm{m}} \omega_{\left(\begin{array}{l}
\| \\
\perp
\end{array}\right)}(T)}{L_{\left(\begin{array}{l}
\| \\
\perp
\end{array}\right)} \gamma_{\left(\begin{array}{l}
\| \\
\perp
\end{array}\right)}(\omega, T)},
$$

with $\gamma_{\left(\begin{array}{l}\| \\ \perp\end{array}\right)}$ taken at $\omega=\omega_{\left(\|_{\perp}\right)}$. The contribution of nonresonant frequencies is so small that their input into the scattering cross section can be neglected.

In the case of MN having a spherical shape $L_{\|}=L_{\perp}=1 / 3$, equations (5.7)-(5.9) become as follows:

$$
\begin{gathered}
\xi_{\mathrm{m}}=\frac{3 \epsilon_{\mathrm{m}}}{2 \epsilon_{\mathrm{m}}+1}, \\
\omega_{\left(\begin{array}{c}
\| \\
\perp
\end{array}\right)}^{2}(T) \equiv \omega_{\mathrm{sph}}=\frac{\omega_{\mathrm{pl}}^{2}(T)}{2 \epsilon_{\mathrm{m}}+1}, \\
\gamma_{\left(\begin{array}{l}
\| \\
\perp
\end{array}\right)}(\omega, T) \equiv \gamma_{\mathrm{sph}}(\omega, T)=\frac{2 \pi}{2 \epsilon_{\mathrm{m}}+1} \sigma(\omega, T) .
\end{gathered}
$$

Then, the expressions (5.3) and (5.4) can be easily transformed to the forms

$$
\begin{aligned}
& C_{\mathrm{abs}}(\omega, T)=9 V \varepsilon_{\mathrm{m}}^{3 / 2} \frac{\omega}{c} \frac{\epsilon^{\prime \prime}(\omega, T)}{\left[\epsilon^{\prime}(\omega, T)+2 \epsilon_{\mathrm{m}}\right]^{2}+\left[\epsilon^{\prime \prime}(\omega, T)\right]^{2}}, \\
& C_{\mathrm{sca}}(\omega, T)=\frac{3 V^{2}}{2 \pi} \epsilon_{\mathrm{m}}^{2}\left(\frac{\omega}{c}\right)^{4} \frac{\left[\epsilon^{\prime}(\omega, T)-\epsilon_{\mathrm{m}}\right]^{2}+\left[\epsilon^{\prime \prime}(\omega, T)\right]^{2}}{\left[\epsilon^{\prime}(\omega, T)+2 \epsilon_{\mathrm{m}}\right]^{2}+\left[\epsilon^{\prime \prime}(\omega, T)\right]^{2}},
\end{aligned}
$$


where we have combined equations (5.11)-(5.13) with the real and imaginary parts of equation (4.7). The most intense cross-sections are observed at the frequency which corresponds to the plasmon resonance of a spherical nanoparticle in the vacuum. Formulae (5.15) and (5.14) yield a resonance when $\epsilon^{\prime}(\omega, T)$ $=-2 \epsilon_{\mathrm{m}}$. The absorption and the scattering cross-sections defined by equations (5.3) and (5.4) reach the maximal values at the frequencies of the SPRs $\omega=\omega_{\left(\begin{array}{l}\| \\ 1\end{array}\right)}$ as well. Near the surface, plasmon resonance light may interact with the particle (at low temperatures) over a cross-sectional area usually larger than the geometric cross section of the particle because the polarizability of the particle becomes very high in this frequency range.

We calculate the scattering efficiency of the MN, which is defined as the ratio

$$
S_{\text {ef }}=\frac{C}{C_{\text {geom }}}, \quad C_{\text {geom }}=\pi\left(\frac{c}{\omega_{\mathrm{pl}}}\right)^{2},
$$

where $C_{\text {geom }}$ is the geometrical cross-section of an individual particle and $C$ is defined by equations (5.3) and (5.4), or by (5.14) and (5.15).

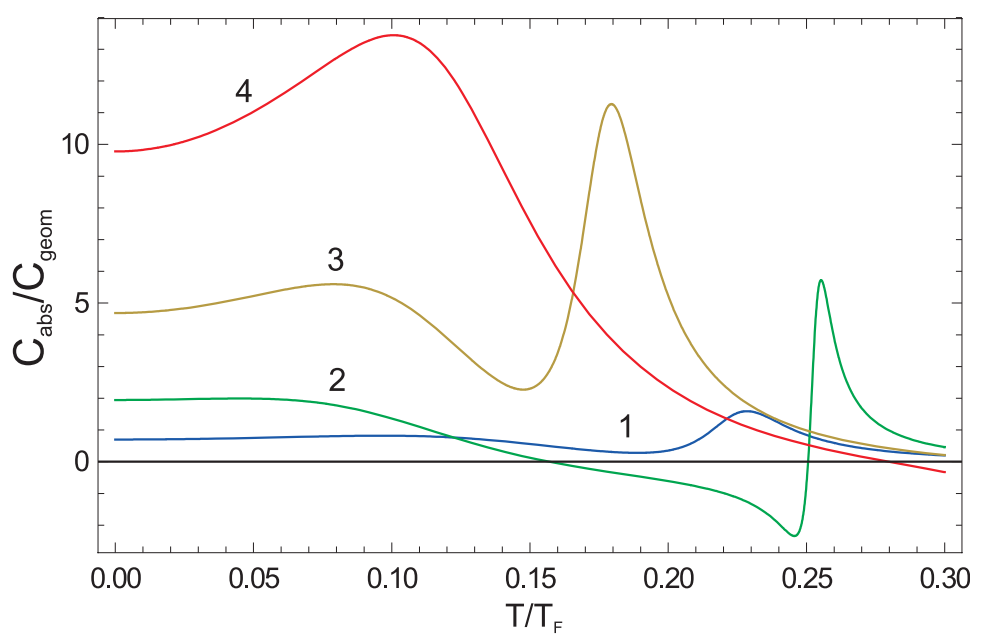

Figure 2. (Color online) The absorption crossection vs temperature for spherical Au particles with different $R(\AA)$ : 78 (1), 100 (2), 125 (3), and 150 (4), embedded in water.

Figure 2 shows the variation of an absorption efficiency as a function of temperature for gold nanoparticles having a spherical shape embedded in water $(n=1.33)$. The calculations were performed in accordance with equations (5.16) and (5.14), with the use of the same parameters as above for figure 1 . For zero temperature, we get the classical result [1, 2], when the SPR intensity depends on the particle radius: larger particles have a larger scattering or absorption cross sections. The situation drastically changes at finite electron temperatures. As we can see from figure 2 the absorption efficiency not only increases (right hand sides of curves 1-4) with the temperature drop, but can also decrease (left hand sides of curves 1-4). We have an interesting situation when the absorption crossection with the temperature lowering increases at first, reaches its peak, then decreases, and starts to increase again. These observations are explained by the conductivity of $\mathrm{MN}$, which oscillate with the particle radius changing [46].

In the case of light scattering (see figure 3), the effect of MN size is more pronounced because the scattering cross-section is proportional to the square of the particle volume.

In figure 3 one can see that the efficiency of light scattering at a resonance frequency does not only increase (curves 1-3) with the growth of the MN radius, but can also decrease (curve 4). The latter means that the efficiency of scattering at a resonance frequency can be suppressed with the temperature rise for some particle radii. Depending on the electron temperature, the scattering efficiency reaches the maximum value, whose peak position depends on the nanoparticle radius.

The peak positions of the resonance plasmon absorption (or scattering) by Au nanoparticle embedded in water can be shifted toward shorter wavelengths as the temperature of electron gas is lowered 


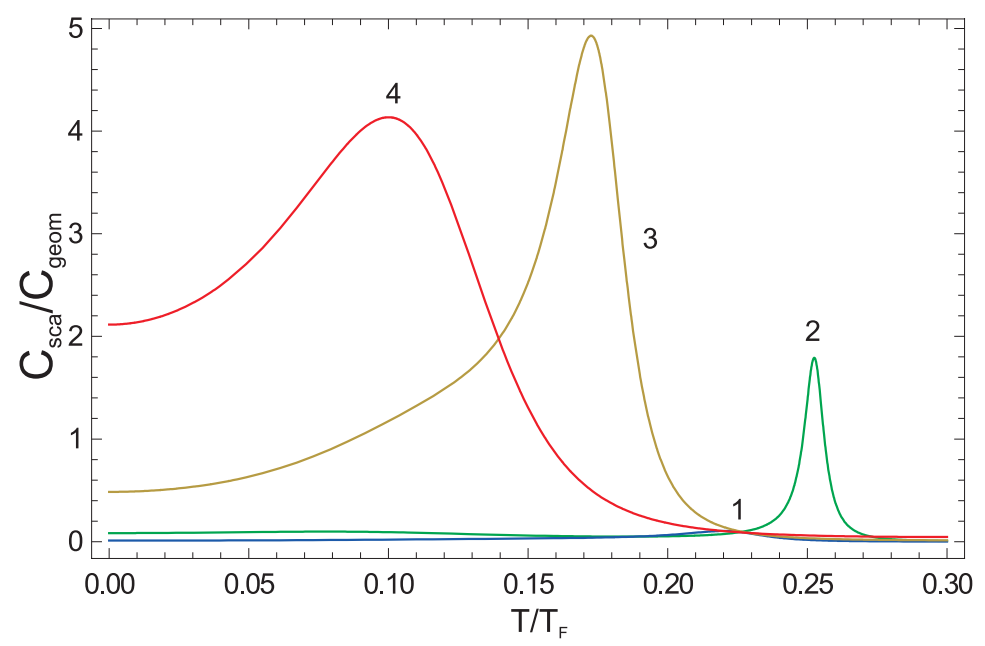

Figure 3. (Color online) The same as in figure2 but for scattering crossection.

after an initial excitation. This behavior is consistent with the known temperature-dependent shift of the differential transmission peak in an isolated sodium nanoparticle [26].

\section{Discussion of results}

Here, we shortly discuss the effects caused by deviations from the spherical particle shape. When the symmetry of the particle decreases, the number of resonance peaks increases. If the shape of a particle deviates from the spherical one, the 3-fold degeneracy dipole mode splits into two (for spheroid) or three (for ellipsoid) modes giving rise to corresponding scattering peaks. In this case, the plasmon response strongly depends on the particle position relative to the illumination direction. To evaluate the electron temperature effect on the absorption or scattering crossections for various illumination directions, one can perform numerical calculations using equations (5.3)-(5.9) and (3.19).

The particle absorption or scattering effects depend on the size and shape of individual particles. Metallic particles that are much smaller than the wavelength of light tend to absorb still more and hence the absorption dominates. The scattered light intensity from small MNs is extremely low. Indeed, Rayleigh scattering theory predicts that the scattering cross section of a spherical cluster should drop as the sixth power of the diameter.

The quantity that is measured experimentally is frequency-resolved time-dependent transmission [26] $\Upsilon_{\text {on }}$ after the excitation of the MN by a pump laser pulse. Then, the static equilibrium transmission $\Upsilon_{\text {off }}$ of the sample measured in the absence of the pump is subtracted to obtain the relative differential transmission [19] $\delta \Upsilon / \Upsilon=\left(\Upsilon_{\text {on }}-\Upsilon_{\text {off }}\right) / \Upsilon_{\text {off. }}$. In the thermal equilibrium, the $\delta \Upsilon$ can be related to the temperature dependent absorption cross section by [37]

$$
\frac{\delta \Upsilon}{\Upsilon}=-\frac{3}{2 \pi R^{2}}\left[C_{\mathrm{abs}}(\omega, T)-C_{\mathrm{abs}}\left(\omega, T_{0}\right)\right]
$$

where $T_{0}$ is the temperature of the $\mathrm{MN}$ before excitation. This relation holds for very small sizes of $\mathrm{MN}$ when the reflectivity of the MN is very small.

A direct comparison of theoretical results with most of the available experimental measurements of the optical properties of MNs is still a matter of debate because inhomogeneities in nanoparticle size, shape, and local environment hide the homogeneous width of the surface plasmon resonance. 


\section{Conclusions}

We have applied a kinetic theory to the calculation of the optical properties of a metal nanoparticle embedded in a dielectric media at different temperatures. It permits to determine the cross-section of light absorption or scattering for various polarizations of the incident electromagnetic wave. The obtained analytical formulas provide the evaluation of the dynamics of light absorption or scattering intensities at the plasmon frequencies for nanoparticles with different radii and shapes, when the temperature of electron gas becomes settled after the action of a laser pulse.

The analytical expressions for electroconductivity and polarizability tensors have been obtained for the case of MNs with a spherical shape. They permit to calculate a number of other physical quantities (e.g., resonance linewidth, figure of merit, etc.) for a particular temperature. We theoretically studied the variation of both the linewidth of SPR and the efficiency of the light absorption and scattering by MN with the change of the electron temperature. The case where the frequency of a laser beam is close to surface plasmon frequencies of a spheroidal $\mathrm{MN}$ is studied in detail.

The high sensitivity of the temperature dependence of SPR linewidth to the radius of a particle was established. Even a small variation in the particle radius can drastically change the run of the SPR linewidth curve vs temperature. This is due to the linewidth oscillations when the radius of MN is varied. The efficiencies of light absorption and scattering for various temperatures strongly depend on the particle radius as well. The Au nanoparticles with different radii are used for illustration.

\section{Acknowledgement}

The author is indebted to Prof. P.M. Tomchuk for helpful discussions and useful comments.

\section{References}

1. Bohren C.F., Huffman D.R., Absorption and Scattering of Light by Small Particles, Wiley, Weinheim, 2004.

2. Kreibig U., Vollmer M., Optical Properties of Metal Clusters, Springer Verlag, Berlin, Heidelberg 1995.

3. Nie S., Emory S.R., Science, 1997, 275, 1102; doi 10.1126/science.275.5303.1102

4. Schroter U., Dereux A., Phys. Rev. B, 2001, 64, 125420; doi 10.1103/PhysRevB.64.125420

5. Hubert C., Rumyantseva A., Lerondel G., Grand J., Kostcheev S., Billot L., Vial A., Bachelot R., Royer P., Nano Lett., 2005, 5, 615; doi 10.1021/nl047956i

6. Fort E., Gresillon S., J. Phys. D: Appl. Phys., 2008, 41, 013001; doi 10.1088/0022-3727/41/1/013001

7. Jung J., Sondergaard T., Bozhevolnyi S.I., Phys. Rev. B, 2007, 76, 035434; doi 10.1103/PhysRevB.76.035434

8. Huang X., Jain P.K., El Sayed I.H., El Sayed M.A., Nanomedicine, 2007, 2, 681; doi 10.2217/17435889.2.5.681

9. Hartland G.V., Ann. Rev. Phys. Chem., 2006, 57, 403; doi 10.1146/annurev.physchem.57.032905.104533.

10. Cai W., White J.S., Brongersma M.L., Nano Lett., 2009, 9, 4403; doi 10.1021/nl902701b

11. Challener W.A., Peng C., Itagi A.V., Karns D., Peng W., Peng Y., Yang X.M., Zhu X., Gokemeijer N.J., Hsia Y.-T., Ju G., Rottmayer R.E., Seigler M.A., Gage E.C., Nat. Photonics, 2009, 3, 220; doi 10.1038/nphoton.2009.26

12. Lowery A., Gobin A., Day E., Halas N., West J., Int. J. Nanomedicine, 2006, 1, 149; doi 10.2147/nano.2006.1.2.149

13. Cao L., Barsic D.N., Guichard A.R., Brongersma M.L., Nano Lett., 2007, 7, 3523; doi 10.1021/nl0722370

14. Lee C.E., Atkins R.A., Taylor H.F., Opt. Lett., 1988, 13, 1038; doi 10.1364/OL.13.001038

15. Yang J.M., Yang H., Lin L., ACS NANO, 2011, 5, 5067; doi 10.1021/nn201142f

16. Kinast J., Turlapov A., Thomas J.E., Chen Q., Stajic J., Levin K., Science, 2005, 307, 1296; doi $10.1126 /$ science.1109220

17. Grigorchuk N.I., Tomchuk P.M., Phys. Rev. B, 2011, 84, 085448; doi 10.1103/PhysRevB.84.085448

18. Kreibig U., J. Phys. F: Metal Phys., 1974, 4, 999; doi 10.1088/0305-4608/4/7/007

19. Bigot J.-Y., Merle J.-C., Cregut O., Daunois A., Phys. Rev. Lett., 1995, 75, 4702; doi 10.1103/PhysRevLett.75.4702

20. Perner M., Bost P., Lemmer U., von Plessen G., Feldmann J., Becker U., Mennig M., Schmitt M., Schmidt H., Phys. Rev. Lett., 1997, 78, 2192; doi 10.1103/PhysRevLett.78.2192

21. Link S., El-Sayed M.A., J. Phys. Chem. B, 1999, 103, 4212; doi 10.1021/jp984796o

22. Florous N.J., Saitoh K., Koshiba M., Opt. Lett., 2006, 31, 404; doi 10.1364/OL.31.000404

23. Florous N.J., Saitoh K., Koshiba M., IEEE Trans. Nanotechnol., 2007, 6, 549; doi 10.1109/TNANO.2007.901187

24. Florous N.J., Saitoh K., Koshiba M., IEEE Photonics Technol. Lett., 2006, 18, 1663; doi 10.1109/LPT.2006.879543

25. Florous N.J., Saitoh K., Koshiba M., IEEE Photonics Technol. Lett., 2007, 19, 324; doi 10.1109/LPT.2007.891618. 
26. Weick G., Weinmann D., Ingold G.-L., Jalabert R.A., Eur. Phys. Lett., 2007, 78, 27002; doi $10.1209 / 0295-5075 / 78 / 27002$

27. Ekici O., Harrison R.K., Durr N.J., Eversole D.S., Lee M., Ben-Yakar A., J. Phys. D: Appl. Phys., 2008, 41, 185501; doi 10.1088/0022-3727/41/18/185501

28. Liu M., Pelton M., Guyot-Sionnest P., Phys. Rev. B, 2009, 79, 035418; doi 10.1103/PhysRevB.79.035418

29. Biehs S.-A., Huth O., Rüting F., Holthaus M., Preprint $\operatorname{arXiv:1103.4511v1.2010.}$

30. Yeshchenko O.A., Bondarchuk I.S., Gurin V.S., Dmitruk I.M., Kotko A.V., Surf. Sci., 2012, 608, 275; doi $10.1016 /$ j.susc.2012.10.019

31. Rosei R., Culp C.H., Weaver J.H, Phys. Rev. B, 1974, 10, 484; doi 10.1103/PhysRevB.10.484

32. Winsemius P., van Kampen F.F., Lengkeek H.P., van Went C.G., J. Phys. F: Metal Phys., 1976, 6, 1583; doi $10.1088 / 0305-4608 / 6 / 8 / 017$

33. Rocca M., Moresco F., Valbusa U., Phys. Rev. B, 1992, 45, 1399; doi 10.1103/PhysRevB.45.1399

34. Groeneveld R.H.M., Sprik R., Lagendijk Ad., Phys. Rev. B, 1995, 51, 11433; doi 10.1103/PhysRevB.51.11433

35. Jensen E.T., Palmer R.E., Allison W., Annett J.F., Phys. Rev. Lett., 1991, 66, 492; doi 10.1103/PhysRevLett.66.492

36. Maier M., Wrigge G., Astruc Hoffmann M., Didier P., v. Issendorff B., Phys. Rev. Lett., 2006, 96, 117405; doi 10.1103/PhysRevLett.96.117405

37. Hervieux P.-A., Benabbas A., Halté V., Bigot J.-Y., Eur. Phys. J. D, 2003, 24, 185; doi 10.1140/epjd/e2003-00115-8

38. Kaganov M.I., Lifshitz I.M., Tanatarov L.V., Zh. Eksp. Teor. Fiz., 1956, 31, 232 (in Russian) [Sov. Phys.- JETP, 1957, 4, 173].

39. Ping Y., Hanson D., Koslow I., Ogitsu T., Prendergast D., Schwegler E., Collins G., Ng A., Phys. Plasmas, 2008, 15, 056303; doi 10.1063/1.2844365

40. Riedl S., Sánchez Guajardo E.R., Kohstall C., Altmeyer A., Wright M.J., Denschlag J.H., Grimm R., Bruun G.M., Smith H., Phys. Rev. A, 2008, 78, 053609; doi 10.1103/PhysRevA.78.053609

41. Yannouleas C., Broglia R.A., Ann. Phys. (N.Y.), 1992, 217, 105; doi 10.1016/0003-4916(92)90340-R

42. Landau L.D., Lifshitz E.M., Electrodynamics of Continous Media, Pergamon, New York, 1986.

43. Cercignani C., Theory and Application of the Boltzmann Equation, Scotish Academic, Edinburg, 1975;

44. Alexandrov A.F., Bogdankevich L.C., Rukhadze A.A., Principles of Plasma Electrodynamics, Springer, Berlin, 1984.

45. Berezkina S.V., Kuznetsova I.A., Yushkanov A.A., Zh. Tekh. Fiz., 2004, 74, No. 12, 67.

46. Grigorchuk N.I., J. Appl. Phys., 2012, 112, 064306; doi 10.1063/1.4751020

47. Levich V.G., A Course of Theoretical Physics, Vol. 1, Nauka, Moskow, 1969 (in Russian).

48. Grigorchuk N.I., J. Phys. Chem. C, 2012, 116, 23704; doi 10.1021/jp3067074

49. Becker J., Trügler A., Jakab A., Hohenester U., Sönnichsen C., Plasmonics, 2010, 5, 161; doi 10.1007/s11468-010-9130-2.

50. Kittel Ch., Introduction to Solid State Physics, Wiley, New York, 1974.

51. Grigorchuk N.I., Eur. Phys. Lett., 2012, 97, 45001; doi 10.1209/0295-5075/97/45001. 


\section{Температурна залежність плазмонних резонансів у сфероїдальних металевих наночастинках}

\section{M.I. Григорчук}

Інститут теоретичної фізики ім. М.М. Боголюбова НАН України, вул. Метрологічна, 14-6, 03680 Київ, Україна

В рамках кінетичної теорії вивчається вплив температури електронів на поглинання та розсіювання світла металевими наночастинками (МH) при збудженні електронних коливань поверхневого плазмона. Одержані формули для тензорів електропровідності та поляризованості для кінцевих температур електронного газу. Детально вивчені електрична провідність та напівширина резонансу поверхневого плазмона для сферичної МН. Досліджується ефективність поглинання та розсіювання світла при зміні температури в залежності від розмірів МН. Зокрема, знайдено, що ефективність поглинання може не тільки збільшуватися при зменшенні температури, а також й зменшуватись. Одержані формули дозволяють аналітично обчислювати різноманітні оптичні й транспортні явища для МН довільної сфероїдальної форми, що знаходяться в довільному діелектричному середовищі.

Ключові слова: електронна температура, металеві наночастинки, електропровідність, тензор поляризованості, резонанс поверхневого плазмона 